\title{
How Well Do Current Laboratory Biomarkers Inform Clinical Decision-Making in Chronic Pain Management?
}

\author{
Jonathan M Hagedorn (iD' \\ Joshua Gunn ${ }^{2}$ \\ Ryan Budwany ${ }^{3}$ \\ Ryan S D'Souza (D) \\ Krishnan Chakravarthy ${ }^{4}$ \\ Timothy R Deer $\mathbb{D i D}^{5}$ \\ 'Department of Anesthesiology and \\ Perioperative Medicine, Division of Pain \\ Medicine, Mayo Clinic, Rochester, MN, \\ USA; ${ }^{2}$ Ethos Research \& Development, \\ Newport, KY, USA; ${ }^{3}$ West Virginia \\ University, Charleston, WV, USA; \\ ${ }^{4}$ University of California - San Diego, San \\ Diego, CA, USA; ${ }^{5}$ The Spine \& Nerve \\ Centers of the Virginias, Charleston, \\ WV, USA
}

Objective: Decision-making in chronic pain patients involves a combination of subjective and objective criteria, including patient history, physical examination, imaging, and patient response to prior treatments, clinical experience, probabilities, and recognition of patterns. However, there is a distinct lack of objective laboratory biomarkers in use in routine clinical care. The objective was to review the literature to identify and describe specific biomarkers in chronic pain management.

Methods: This is a narrative review of the literature regarding the use of laboratory biomarkers in chronic pain. A librarian-assisted literature search of the PubMed, Science Direct, and Google Scholar databases was performed and resulted in 304 possible manuscripts. We included manuscripts assessing laboratory collected biomarkers from urine, serum, cerebrospinal fluid, and saliva. After screening and review of the initial literature search results, a total of 75 manuscripts were included in the narrative review.

Conclusion: The studies reviewed suggested that specific biomarkers may help identify those patients at risk of disease development and function as a prognostic indicator for disease progression and treatment response. However, additional research is necessary before specific recommendations can be made, and current clinical decision-making is modified.

Keywords: chronic pain, biomarkers, outcomes

\section{Introduction}

Clinical decision-making in chronic pain patients involves a combination of subjective and objective criteria, including patient history, physical examination, medical imaging, and patient response to prior treatments, coupled with clinical experience, probabilities, and recognition of patterns (Table 1). In other areas of medicine, specific objective data (ie, blood pressure, lipid panel, blood glucose, etc) can be collected and analyzed, both for diagnostic purposes and assessing treatment response. For pain physicians, to date, this diagnostic option has not been available. Instead, pain management teams have relied on pain rating scales (eg, visual analog scale [VAS], numeric rating scale [NRS], patient-reported pain relief, among others), which are easily manipulated and not interchangeable.

Fortunately, researchers have been searching for pain-specific objective criteria and laboratory evaluations, and this includes biomarkers. Biomarkers are used as an indicator of normal and pathologic processes, therapeutic response to an intervention, identify individuals at risk of disease development, and to certify the diagnosis. Ideally for chronic pain, the biomarkers would be direct correlates to the
Correspondence: Timothy R Deer The Spine \& Nerve Centers of the Virginias, Charleston, West Virginia, 2530I, USA

Tel + I 304-347-6/20

Email doctdeer@aol.com 
Table I Traditional Clinical Decision-Making Pathway

\begin{tabular}{|l|r|}
\hline Diagnostic Variable & Examples \\
\hline Patient history & $\begin{array}{r}\text { Modified depending on site of pain; may include palpation, range of motion, sensory and motor testing, and specific } \\
\text { maneuvers (eg, facet loading, straight leg raise, FABER test, etc) }\end{array}$ \\
\hline $\begin{array}{l}\text { Physical examination } \\
\text { motor changes }\end{array}$ \\
\hline Medical imaging & X-ray, computerized tomography (CT) scan, magnetic resonance imaging (MRI) \\
\hline Specialty tests & Electromyography (EMG), quantitative sudomotor axon reflex test (QSART) \\
\hline $\begin{array}{l}\text { Patient response to prior } \\
\text { treatments }\end{array}$ & Documented pain relief with length of time of past treatments \\
\hline
\end{tabular}

underlying biologic processes involved in chronic pain creation versus the subjective chronic pain measurements, including oxidative stress, systemic inflammation, nutritional status, and/or neurotransmitter creation and destruction. In doing so, treatments could become more targeted to the causative chronic pain mechanism.

Initial chronic pain biomarkers have been sought in laboratory studies, in addition to quantitative sensory testing, skin biopsy, and brain imaging. ${ }^{1}$ This review will focus on laboratory collected biomarkers, including urinary, serum, cerebrospinal fluid (CSF), and salivary studies.

\section{Methods}

This is a narrative review of the literature regarding the use of laboratory biomarkers in chronic pain. The objective was to review the literature to identify and describe specific biomarkers in chronic pain management. A librarian-assisted literature search of the PubMed, Science Direct, and Google Scholar databases was performed to identify appropriate manuscripts. The terms "chronic pain", "intractable pain", "biomarkers", "blood", "serum", "hematologic tests", "urine", "saliva", "salivary", "cerebrospinal fluid", "spinal fluid", and "biologic" were searched and potential manuscripts were collated. All results were in the English language and no date range was used. We also reviewed former narrative reviews to supplement the literature search. These parameters resulted in collection of 304 potential manuscripts (Figure 1). Manuscripts were included based on the level of evidence, quality of results obtained, impact of the conclusions, and if the study allowed increased breadth of the topic. Manuscripts were excluded if they did not relate to the subject matter. After removal of duplicates and screening the abstracts of the initial literature search results, a total of 85 manuscripts underwent full-text review. Following full-text review, 75 manuscripts were included in the manuscript.

\section{Literature Review of Chronic Pain Biomarkers Urinary Biomarkers}

Urinary biomarkers of acute and chronic pain states are of tremendous interest to researchers due to the non-invasive nature of urine collection and the relative simplicity of urine as a biological matrix for analysis. Table 2 lists the most studied urinary biomarkers. ${ }^{2-38}$ Urine contains cellular elements, biochemicals, metabolites, and proteins derived from glomerular filtration of plasma, and renal tube excretion that provide valuable insight into the metabolic and pathophysiological state of an individual. ${ }^{38}$ Biomarker analysis in urine also affords certain analytical advantages over serum or plasma due to the concentrating ability of the kidneys, which often leads to higher levels of target analytes. Clinical utility of validated pain biomarkers is anticipated to range from diagnostic enrichment to predicting drug and/or interventional therapy efficacy in individual patients. Of particular interest to researchers and clinicians is the validation of actionable pain biomarkers, which could be monitored longitudinally to provide objective documentation of improvement, worsening, or response to a therapy. For such applications, urine represents the most highly desirable biospecimen due to the non-invasive collection techniques that can be conducted recurrently without specialized training, patient instruction, and/or expense.

High-throughput 'omics' technologies, facilitating the rapid exploration of genomic, proteomic, transcriptomic, and metabolomic data, has greatly facilitated the search of 


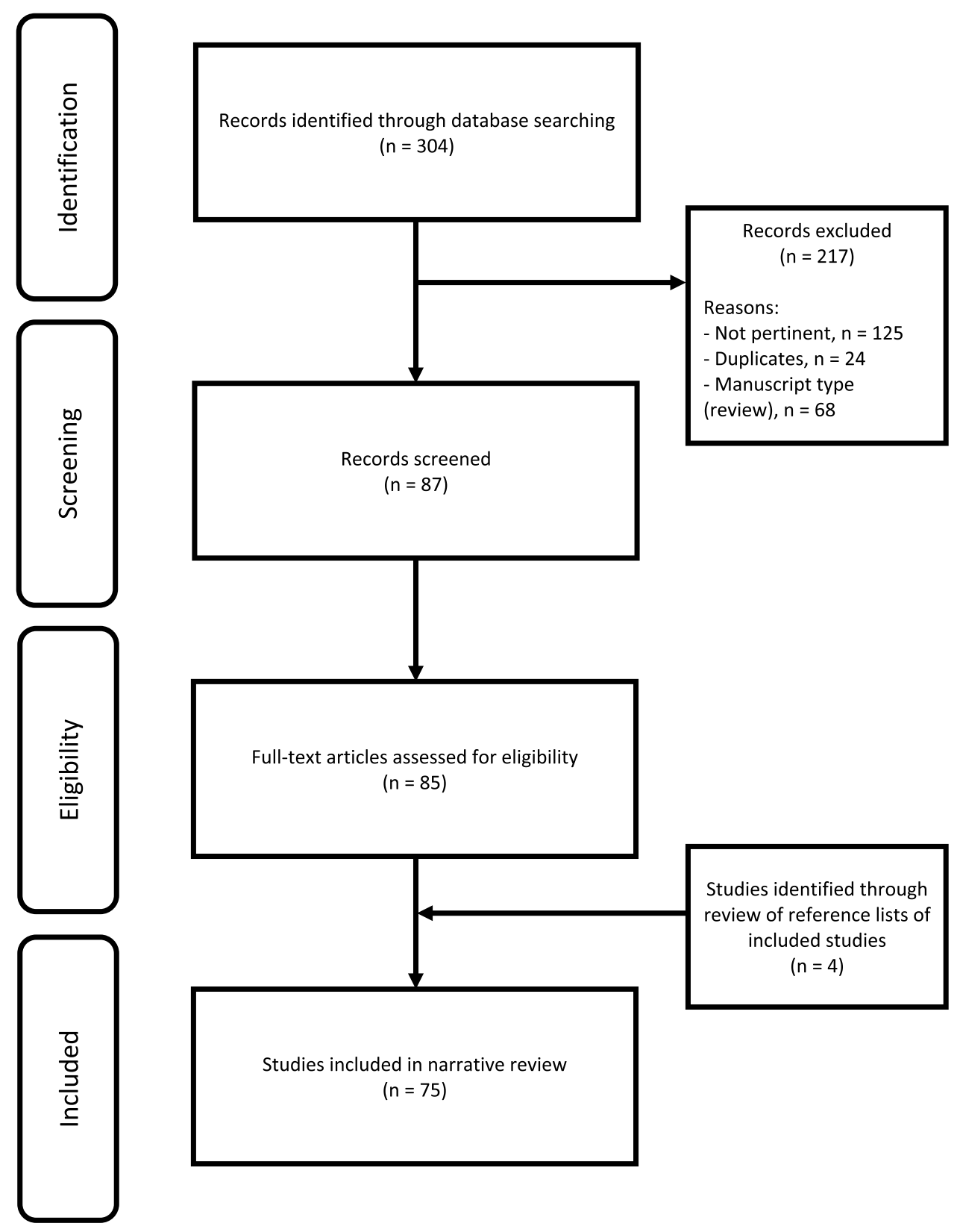

Figure I Flow Diagram of Screening and Selection Process.

clinically meaningful biomarkers in recent years. While much of the pain biomarker research utilizing CSF, serum, and plasma has focused on genomic, proteomic, and/or transcriptomic targets, ${ }^{39-43}$ much of the pioneering work involving urine has focused on metabolomic targets. ${ }^{44-46}$ Deer et al detailed the findings of a retrospective, observational study, which evaluated the prevalence of abnormal metabolomic biomarker findings in a cohort of 17,834 chronic pain patients. This study utilized a predefined, targeted urine panel comprising 11 endogenous biomarkers of systemic inflammation, oxidative stress, neurotransmitter metabolism, and micronutrient deficiencies. Composite biomarkers of this unique panel all represent biochemical pathways known to impact the development or worsening of pain. Interestingly, each of the pain-pathways described in the study may also be "druggable" or modulated with existing treatment options such that abnormal biomarker findings could be corrected with existing compounds. The authors describe the panel as a cost-effective way to identify biochemical drivers of pain, which would afford safe, targeted, non-opioid therapy options. Findings of the retrospective analysis revealed that $77 \%$ of chronic pain 
Table 2 Urinary Biomarkers and Relevance in Chronic Pain Pathology

\begin{tabular}{|c|c|c|}
\hline Biomarker & Relevance & References \\
\hline Methylmalonic acid & $\begin{array}{l}\text { Elevated with vitamin } \mathrm{B} 12 \text { deficiency. } \\
\text { Vitamin } \mathrm{B} 12 \text { deficiency leads to nerve damage and degeneration of the spinal cord. } \\
\text { Peripheral neuropathy is the most common pain presentation. }\end{array}$ & {$[2-5]$} \\
\hline Xanthurenic acid & $\begin{array}{l}\text { Elevated levels indicate a vitamin B6 deficiency. } \\
\text { Neuropathy to due to vitamin B6 deficiency starts with numbness, paresthesias, or } \\
\text { burning pain in the feet, which then ascends to affect the legs and hands. }\end{array}$ & [5-9] \\
\hline Homocysteine & $\begin{array}{l}\text { Elevated levels commonly indicate a B-vitamin (B6/B9/B/2) deficiency. } \\
\text { Elevated homocysteine results from B-vitamin deficiencies. Elevated homocysteine } \\
\text { levels cause inflammation by increasing arachidonic acid and the proinflammatory } \\
\text { prostaglandin E2 production. }\end{array}$ & {$[10-12]$} \\
\hline 3-HPMA & $\begin{array}{l}\text { Elevated levels indicate increased exposure to acrolein. } \\
\text { Acrolein contributes to inflammatory pain sensitivities through its binding and } \\
\text { activation of the transient receptor potential ankyrin I receptor (TRPAI) in nerve } \\
\text { fibers. }\end{array}$ & {$[13-15]$} \\
\hline Quinolinic acid & $\begin{array}{l}\text { Elevated levels indicate cytokine-mediated chronic inflammation. } \\
\text { Quinolinic acid is a neuroactive kynurenine pathway (KP) metabolite which serves as } \\
\text { a sensitive marker of chronic, systemic inflammation. Upregulation of this pathway } \\
\text { has been shown to play a central role in the comorbidity of pain and depression. } \\
\text { Quinolinic acid induces its depressive effects through its action on NMDA receptors. }\end{array}$ & {$[16-20]$} \\
\hline Kynurenic acid & $\begin{array}{l}\text { Elevated levels indicate cytokine-mediated chronic inflammation. } \\
\text { Kynurenate is a neuroactive kynurenine pathway }(\mathrm{KP}) \text { metabolite which serves as } \\
\text { a sensitive marker of chronic, systemic inflammation. Upregulation of this pathway } \\
\text { has been shown to play a central role in the comorbidity of pain and depression. }\end{array}$ & {$[16-20]$} \\
\hline Pyroglutamate & $\begin{array}{l}\text { Elevated levels indicate glutathione depletion. } \\
\text { Glutathione depletion renders nerve cells susceptible to oxidative damage, which can } \\
\text { lead to neuropathic pain. }\end{array}$ & {$[21-23]$} \\
\hline Hydroxymethylglutarate & $\begin{array}{l}\text { Elevated levels indicate a Coenzyme Q10 deficiency. } \\
\text { Coenzyme Q10 deficiencies can cause muscle weakness and pain. }\end{array}$ & {$[24,25]$} \\
\hline Ethylmalonate & $\begin{array}{l}\text { Elevated levels indicate a carnitine deficiency. } \\
\text { Carnitine deficiencies cause muscle aches and fatigue. }\end{array}$ & {$[26,27]$} \\
\hline 5-Hydroxyindoleacetate (5-HIAA) & $\begin{array}{l}\text { Abnormally low levels indicate decreased synthesis/turnover of serotonin. } \\
\text { Abnormally low synthesis/turnover of serotonin can heighten pain sensitivity. }\end{array}$ & [28-30] \\
\hline Vanilmandelate & $\begin{array}{l}\text { Abnormally low levels indicate decreased synthesis/turnover of norepinephrine. } \\
\text { Abnormally low synthesis/turnover of norepinephrine can heighten pain sensitivity. }\end{array}$ & {$[31,32]$} \\
\hline Prostatic exosomal proteins (PSEP) & $\begin{array}{l}\text { Secreted by prostate corpuscles. } \\
\text { Elevated PSEP was found to correlate with chronic prostatitis and chronic pelvic pain } \\
\text { syndrome symptoms. }\end{array}$ & {$[33,34]$} \\
\hline $\begin{array}{l}\text { Matrix metalloproteinase (MMP), including } \\
\text { MMP-9, MMP-9/NGAL, MMP-2 }\end{array}$ & $\begin{array}{l}\text { Involved with tissue remodeling and extracellular matrix degradation. Moderate key } \\
\text { cellular processes, including angiogenesis, cytokine release, and inflammation. } \\
\text { Studied in urological chronic pelvic pain syndrome and have been shown to positively } \\
\text { correlate with severity of pain and symptoms in women with this pathology. }\end{array}$ & [35-38] \\
\hline $\begin{array}{l}\text { Neutrophil gelatinase-associated lipocalin } \\
\text { (NGAL; also known as LCN 2) }\end{array}$ & $\begin{array}{l}\text { Released by neutrophils in response to tissue inflammation and infection. } \\
\text { Studied in urological chronic pelvic pain syndrome. }\end{array}$ & {$[35]$} \\
\hline
\end{tabular}


Table 2 (Continued).

\begin{tabular}{|l|l|l|}
\hline Biomarker & Relevance & References \\
\hline $\begin{array}{l}\text { Vascular endothelial growth factor (VEGF, } \\
\text { VEGF-RI) }\end{array}$ & $\begin{array}{l}\text { Plays important role in modulating pain. Also involved with vascular permeability and } \\
\text { angiogenesis. } \\
\text { Studied in urological chronic pelvic pain syndrome and positively correlate with pain } \\
\text { severity. }\end{array}$ & {$[34,36,37]$} \\
\hline
\end{tabular}

subjects exhibited at least one abnormal biomarker finding. ${ }^{44}$

In the same year, authors of the aforementioned study completed a prospective, multi-site, cross-sectional study aimed at establishing the clinical validity of this 11biomarker urine panel known as the Foundation Pain Index (Foundation PI). ${ }^{45}$ Clinical validation of Foundation PI was established in a prospective, crosssectional study designed to evaluate the criterion, discriminant, and face validity of this novel test offering in a population of chronic pain subjects. Studies confirmed that this multi-analyte assay with algorithmic analysis reveals novel objective data to guide targeted, non-opioid treatment strategies, including a number of different vitamins to treat underlying pathologies. Additionally, Foundation PI scores strongly segregate biomarker profiles of chronic pain subjects from pain-free controls and correlate with worsening clinical assessments of chronic pain. This information has not been previously available and provides objective, mechanistic insight into the pathology of chronic pain in a diverse population of chronic pain subjects.

Criterion validation analysis evaluated the significance of correlations between Foundation PI scores and validated clinical assessments for chronic pain. The 36-Item Short-Form Survey (SF-36) was selected as the most appropriate validated clinical assessment to perform criterion validation analysis as it evaluates both the physical and emotional impact of pain on subjects and is widely used in clinical practice. In a cohort of 153 chronic pain subjects and 334 age-and sex-matched pain-free controls, Foundation PI scores were significantly associated with overall SF-36 scores $(P=0.041)$ as well as with individual components of the SF-36, including general health $(P=$ $0.0457)$, emotional well-being $(P=0.0044)$, and limitations due to emotional problems $(P=0.0011){ }^{45}$ Correlations of Foundation PI scores with both physical and emotional components of the SF-36 are not surprising considering the diverse set of biochemical and metabolic pathways represented by this biomarker set. Strong validation performance of Foundation PI indicates a central role for deranged biochemistry in the etiology of chronic pain, which is incredibly encouraging due to the reversible nature of biomarker abnormalities detected by this novel assay.

McCloy and colleagues conducted a comprehensive systematic review of the association between urinary biomarkers and pain, which was published as part of the International Federation for Medical and Biological Engineering (IFMBE) book series. ${ }^{47}$ The authors reviewed findings from 277 studies comprising 22 randomized controlled trials (RCTs), 44 controlled observational studies, and 211 case studies or series. The RCTs involved 2177 patients with various pain diagnoses in which urinary biomarkers were measured as part of the study. The authors conclude that urinary biomarkers in painful conditions of bone turnover have consistently found statistically significant correlations between disease progression and pain but caution that chosen biomarkers may reflect the disease mechanism rather than pain levels.

Liang et al recently described the development of a urine-based biomarker for the detection of symptomatic progression of chronic prostatitis/chronic pelvic pain syndrome (CP/CPPS) in a retrospective multi-center study utilizing prostatic exosomal proteins (PSEPs) levels. ${ }^{33}$ Spearman correlation coefficient showed a significant level of correlation between the NIH-Chronic Prostatitis Symptom Index (NIH-CPSI) and the urinary PSEP level ( $\mathrm{rs}=0.194, \mathrm{P}=0.0035$ ). The authors conclude that findings highlight the potential role of PSEP as a practical indicator of the symptomatic progression of CP/CPPS and clinical application of this assay may guide drug discovery and lead to better treatment options and improved quality of life.

In 2017, Dagher et al reported the findings of a large study aimed at comparing candidate Urological Chronic Pelvic Pain Syndrome (UCPPS) biomarkers among UCPPS subjects and control cohorts to gain new insights 
into underlying pathophysiology. ${ }^{37}$ Authors also examined associations of biomarkers with symptom severity within the UCPPS cohort and described the potential role of validated biomarkers in the development of new treatment strategies, as objective measures for patient classification schemes and evaluation of clinical outcomes, as well as further characterization of underlying disease mechanism. Results demonstrated that males with UCPPS had significantly higher normalized protein concentration of vascular endothelial growth factor (VEGF), VEGF receptor-1 (VEGF-R1), and matrix metallopeptidase 9 (MMP-9) than healthy male controls. Furthermore, males with UCPSS had significantly higher concentrations of VEGF than female healthy controls. Additionally, pain and urinary symptoms were found to be positively associated with the MMP-9/NGAL complex in women.

\section{Serum Biomarkers}

The current literature regarding serum biomarkers is shown in Table 3. ${ }^{40,48-64,68}$ De Queiroz et al found that muscle damage and injury were associated with metabolic and inflammatory responses and the biochemical milieu of select inflammatory mediators, neuropeptides, and cytokines were found to be higher in subjects with painful muscle injury compared with those with latent injury or absent any muscle metabolic products (eg, creatine kinase). ${ }^{53}$ Thus, the combination of inflammatory cytokines measured with muscle metabolism biomarkers may be used to differentiate the specific contributions of acute muscle injury to undefined back pain.

Pedersen et al studied the serum levels of Interleukin (IL)-6 (IL-6) and IL-8 in patients with lumbar radicular pain due to disc herniation. ${ }^{55,56}$ Samples were collected at subject recruitment and at 6-weeks or the 12-month follow-up time points, regardless of the treatment they received. They compared serum levels of IL-6 and IL-8 with patient-reported pain intensity on a $10 \mathrm{~cm}$ VAS. In this study, it was found that chronic lumbar radicular pain may be associated with a persistent increase of the proinflammatory cytokines IL-6 and IL- 8 in serum after disc herniation, suggesting that these cytokines may be associated with the mechanisms underlying development of chronic pain due to disc herniation.

Current research surrounding low back pain (LBP) and associated inflammation and biomarkers focuses on chronic presentation of symptoms and the level of symptom severity. Multiple studies have observed significant differences in proinflammatory cytokines (eg, IL-6, tumor necrosis factor-
$\alpha[\mathrm{TNF}-\alpha]$, IL-8, and IL-1 $\beta$ ) in relation to pain intensity. Uçeyler et al compared cytokine profiles of patients with painful versus painless neuropathies and identified higher levels of proinflammatory cytokines in the serum of patients experiencing pain. ${ }^{58}$ Patients were grouped on the basis of severity of patient-reported outcomes of LBP and were found to have significantly higher serum protein levels and serum mRNA levels of IL-6, IL- 8 , and TNF- $\alpha$. Licciardone et al showed that IL- 6 and IL-1 $\beta$ were significantly correlated with both pain severity and somatic dysfunction. $^{64}$ In a study by Wang et al, patients were divided into groups of mild and severe sciatica, and both IL-6 and IL- 8 were found to be greater in severe sciatica patients compared not only with controls but also with mild sciatica patients, suggesting that proinflammatory cytokine levels may contribute to sciatica intensity. ${ }^{65}$

A study by Uçeyler et al showed that anti-inflammatory markers IL-4 and IL-10 were significantly lower in subjects experiencing lesser or no LBP and higher serum mRNA levels of IL-10 and IL-4 were found in painless neuropathy patients. ${ }^{58}$ IL-4 and IL-10 are specific cytokines that are produced by activated macrophages and monocytes and considered to be anti-inflammatory because they can inhibit proinflammatory cytokine synthesis. In further work by Wang et al, elevated levels of IL-4 and IL-10 were found in mild sciatica patients as compared with severe cases. ${ }^{65}$ This may suggest that there are analgesic effects of anti-inflammatory cytokines, which may also indicate a more favorable outcome to treatment.

Through the use of the McGill Pain Questionnaire, De Queiroz et al reported a positive correlation between TNF- $\alpha$ and current pain intensity, as well as "severity of pain in the last week." ${ }^{53}$ Serum protein levels and mRNA expression of TNF- $\alpha$ have been shown to be significantly higher in subjects experiencing greater intensity of chronic LBP. A study by Uçeyler et al showed a twofold higher TNF- $\alpha$ protein levels in patients with painful neuropathies compared to patients with painless neuropathies and healthy controls. ${ }^{58}$ Wang et al showed, in a study that categorized patients by severity of sciatica pain, that those with severe sciatica had twofold higher TNF- $\alpha$ serum levels than those without sciatica and performed a prospective comparative longitudinal study, which found that there was a significantly higher proportion of TNF- $\alpha$ participants in the patient group than in the control group, which was sustained through multiple time points over a 6-month follow-up. ${ }^{65}$ Additionally, they investigated the cross-sectional associations of depressive symptoms as a comorbidity that 
Table 3 Serum Biomarkers and Relevance in Chronic Pain Pathology

\begin{tabular}{|c|c|c|}
\hline Biomarker & Relevance & References \\
\hline High-sensitivity C-reactive protein (hsCRP) & $\begin{array}{l}\text { Acute phase protein. } \\
\text { Positively associated with pain levels in acute LBP profiles. }\end{array}$ & {$[48,49]$} \\
\hline Tumor necrosis factor $\alpha$ (TNF- $\alpha)$ & $\begin{array}{l}\text { Cytokine that stimulates inflammatory responses, induces nerve swelling and } \\
\text { neuropathic pain, and promotes cellular apoptosis via its cytotoxic effect. } \\
\text { Serum protein levels and mRNA expression of TNF- } \alpha \text { have been shown to be } \\
\text { significantly higher in subjects experiencing greater intensity of LBP. }\end{array}$ & {$[50-52]$} \\
\hline Soluble TNF receptor I (sTNFRI) & Positively correlated with measures of pain intensity or severity (acute pain). & [53] \\
\hline Interleukin-6 (IL-6) & $\begin{array}{l}\text { Helps mediate the acute-phase response to injury by promoting monocyte } \\
\text { differentiation into macrophages and activating maturation of lymphocytes (acute } \\
\text { pain). } \\
\text { Exercise-induced injury models have found elevated serum levels of IL-6 released } \\
\text { by injured skeletal muscle. }\end{array}$ & {$[54-57,64]$} \\
\hline Interleukin-4 (IL-4) & $\begin{array}{l}\text { Lower in subjects experiencing lesser or no LBP, may be elevated in those } \\
\text { experiencing higher pain. }\end{array}$ & [58] \\
\hline Interleukin-I0 (IL-10) & $\begin{array}{l}\text { Lower in subjects experiencing lesser or no LBP, may be elevated in those } \\
\text { experiencing higher pain. }\end{array}$ & [58] \\
\hline $\begin{array}{l}\text { T helper } 17 \text { (THI7) cells and Interleukin- I7 } \\
\text { (IL-I7) }\end{array}$ & $\begin{array}{l}\text { Increased levels of THI7 cells and IL-17, leads to inflammation, further } \\
\text { deterioration, and increased pain. }\end{array}$ & {$[59,60]$} \\
\hline $\begin{array}{l}\text { Interleukin-2I (IL-2I), Interleukin-I7 (IL-I7), } \\
\text { and Cyclooxygenase-2 (COX-2) }\end{array}$ & $\begin{array}{l}\text { VAS pain scores were positively correlated with the serum levels. } \\
\text { Higher expression was found in protein mRNA levels in disc tissue for disc } \\
\text { herniation patients. }\end{array}$ & [6I] \\
\hline $\begin{array}{l}\text { Regulated on activation, normal T cell } \\
\text { expressed and secreted (RANTES) }\end{array}$ & $\begin{array}{l}\text { Expressed in response to inflammatory stimuli and leads to catabolic activity. } \\
\text { Higher levels of RANTES are associated with greater impairment and activity } \\
\text { limitation, suggesting a link between inflammation and activity-related disability. }\end{array}$ & [62] \\
\hline $\begin{array}{l}\text { Apolipoprotein-M (APO-M), tetranectin (TN), } \\
\text { and immunoglobulin light chain (IGL) }\end{array}$ & Found to be significantly lower in patients with disc herniation. & [63] \\
\hline Apolipoprotein-LI (APO-LI) & Found to be significantly higher in patients with disc herniation. & [63] \\
\hline Microfibril-associated protein 3 (MFAP3) & Decreased in blood in high pain states. & [40] \\
\hline G protein subunit gamma 7 (GNG7) & $\begin{array}{l}\text { Decreased in blood in high pain states. } \\
\text { Decrease in blood is a strong predictor of future emergency room visits. }\end{array}$ & [40] \\
\hline Contactin I (CNTNI) & Decreased in blood in high pain states. & {$[40]$} \\
\hline Lymphocyte antigen 9 (LY9) & Increased in blood in high pain states. & {$[40]$} \\
\hline $\begin{array}{l}\text { Coiled-coil domain containing I44B } \\
\text { (CCDCI44B) }\end{array}$ & Decreased in blood in high pain states. & {$[40]$} \\
\hline Guanylate binding protein I (GBPI) & Increased in blood in high pain states. & {$[40]$} \\
\hline $\begin{array}{l}\text { Interleukin-8 (IL-8) and Monocyte } \\
\text { chemotactic protein-I (MCP-I) }\end{array}$ & $\begin{array}{l}\text { Increased in blood in fibromyalgia patients and positively correlated with pain } \\
\text { severity. }\end{array}$ & [68] \\
\hline
\end{tabular}

can contribute to levels of chronic LBP and potentially to inflammation, and found that although elevated TNF- $\alpha$ is associated LBP, these levels were not modulated by depression in patients. ${ }^{66,67}$
Through investigating the involvement IL-21, IL-17, and cyclooxygenase-2 (COX-2) in disc herniation, Xue et al demonstrated that disc herniation patients exhibited significantly higher levels of serum IL-21 and IL-17. 
Higher expression of IL-21, IL-17, and COX-2 was found in protein and mRNA levels in disc tissues from disc herniation patients than in normal disc tissues. ${ }^{61}$ They concluded that VAS pain scores, IL-17, and COX-2 were positively correlated with the IL-21 levels, implicating its role in the pathogenesis of lumbar disc herniation.

Regulated on activation, normal $\mathrm{T}$ cell expressed and secreted (RANTES) is expressed in response to inflammatory stimuli and leads to catabolic activity. Sowa et al showed significant associations between levels of RANTES after activity, and pain levels and pain-related functioning. ${ }^{62}$ The study found that higher RANTES levels were correlated with higher affective scores, a measure of pain interpretation. This indicates an important role of this systemic biomarker in the experience of chronic LBP. Furthermore, it was concluded that higher levels of RANTES are associated with greater impairment and activity limitation, suggesting a link between inflammation and activity-related disability.

Xie et al used proteomic analysis of blood samples to establish whether there are serum proteins associated with disc herniation. ${ }^{63}$ Through the use of twodimensional electrophoresis of blood samples from patients and control subjects, distinct protein spots were identified by matrix-assisted laser desorption/ionization time-of-flight mass spectrometry. Results showed that apolipoprotein-L1 (APO-L1), apolipoprotein-M (APO$\mathrm{M})$, tetranectin $(\mathrm{TN})$, and immunoglobulin light chain (IGL) differed in patients with lumbar disc herniation. These investigators found that mean serum concentrations of APO-M, TN, and IGL were significantly lower in patients with disc herniation, whereas levels of APOL1 were significantly higher. Very little is known about these biomarkers and similar to all the others mentioned, more long-term investigation is needed.

Niculescu et al sought to identify objective serum biomarkers for pain in psychiatric patients. ${ }^{40} \mathrm{~A}$ total of 403 subjects were identified from the Indianapolis VA Medical Center and were followed longitudinally resulting in 493 blood samples. The authors reported being successful in discovering gene expression biomarkers that predicted pain and future emergency department visits. The study found that microfibril associated protein 3 (MFAP3) was the strongest predictor for pain, especially in females and males with post-traumatic stress disorder. Additionally, G protein subunit gamma 7 (GNG7), contactin 1 (CNTN1), lymphocyte antigen 9 (LY9), coiledcoil domain containing 144B (CCDC144B), and guanylate-binding protein 1 (GBP1) were also viable markers for those individuals with chronic pain. GNG7 was identified as a strong marker for future emergency room visits for pain.

Interleukin- 8 and monocyte chemotactic protein-1 (MCP-1) were found to be elevated in fibromyalgia patients in a study by Ang et al. ${ }^{68} \mathrm{~A}$ total of 16 patients previously diagnosed with fibromyalgia provided two blood samples (week 1 and week 12) and the changes in IL-8 and MCP-1 concentrations were compared with pain severity at the two visits. The researchers found that the change in pain severity was positively correlated with changes in IL-8 and MCP-1 (ie, as pain severity increased, biomarker concentrations increased). The authors concluded that IL- 8 and MCP-1 may assist in determining prognosis and monitoring treatment response.

\section{Cerebrospinal Fluid Biomarkers}

The list of reported CSF biomarkers is found in Table $4 .{ }^{69-76}$ The CSF contains various proteins, signaling molecules and peptides, metabolites, and other products that are in direct contact with the spinal cord and brain. ${ }^{73}$ Fluctuations in these markers may reflect the biochemical changes in the central nervous system. In fact, the proteomics studies utilizing comprehensive mass spectrometry have reported 2500 to 3000 proteins within the CSF. ${ }^{73,77,78}$ While evidence remains limited, multiple studies have highlighted changes in biomarker levels that may reflect mechanisms related to acute and chronic pain manifestations.

Wahlbeck et al compared levels of angiotensin converting enzyme (ACE) and arginine vasopressin (AVP) between 15 patients with chronic pain disorder and 19 healthy control patients. ${ }^{70}$ Serum AVP levels were significantly higher in chronic pain patients versus healthy controls. Concordant with this observation, serum osmolality which stimulates release of AVP was also elevated in chronic pain patients versus healthy controls. However, there was no significant in CSF ACE level between cohorts.

Brisby et al reported on 39 patients with lumbar disc herniation and sciatica in which CSF and serum concentrations of IL- $1 \beta$, IL- 6 , IL- 8 , interferon (IFN)- $\gamma$ and TNF- $\alpha$ were investigated using the enzyme-linked immunosorbent assay (ELISA) technique. ${ }^{69}$ CSF IL- 8 concentrations were increased in $30.8 \%$ of the patients (12/39) and were correlated with a short duration of pain and disc extrusion or sequestration. The authors hypothesized that the increase in IL-8 is related to mechanical nerve root compression. 
Table 4 Cerebrospinal Fluid Biomarkers and Relevance in Chronic Pain Pathology

\begin{tabular}{|c|c|c|}
\hline Biomarker & Relevance & References \\
\hline Interleukin-8 (IL-8) & Higher concentration is reported in patients with disc herniation. & [69] \\
\hline Arginine vasopressin (AVP) & Higher in chronic pain patients. & [70] \\
\hline $\begin{array}{l}\text { Glial cell line-derived neurotrophic } \\
\text { factor (GDNF) }\end{array}$ & $\begin{array}{l}\text { Reduced expression in patients with fibromyalgia, chronic migraine, and probable } \\
\text { analgesic abuse headache. }\end{array}$ & {$[7 I]$} \\
\hline Somatostatin (SST) & $\begin{array}{l}\text { Reduced expression in patients with fibromyalgia, chronic migraine, and probable } \\
\text { analgesic abuse headache. } \\
\text { Lower expression after burst spinal cord stimulation in patients with neuropathic pain. }\end{array}$ & {$[71,72]$} \\
\hline Neural cell adhesion molecule LI & Higher levels associated with pain in fibromyalgia and rheumatoid arthritis. & [73] \\
\hline Complement C4-A & Higher levels associated with pain in fibromyalgia and rheumatoid arthritis. & [73] \\
\hline Lysozyme C & Higher levels associated with pain in fibromyalgia and rheumatoid arthritis. & [73] \\
\hline $\begin{array}{l}\text { Receptor-type tyrosine-protein } \\
\text { phosphatase zeta }\end{array}$ & Higher levels associated with pain in fibromyalgia and rheumatoid arthritis. & [73] \\
\hline Apolipoprotein-D & Higher levels associated with pain in fibromyalgia and rheumatoid arthritis. & [73] \\
\hline Alpha-I-antichymotrypsin & Higher levels associated with pain in fibromyalgia and rheumatoid arthritis. & [73] \\
\hline Granulins & Higher levels associated with pain in fibromyalgia and rheumatoid arthritis. & [73] \\
\hline $\begin{array}{l}\text { Calcium/calmodulin-dependent protein } \\
\text { kinase type II subunit alpha }\end{array}$ & Higher levels associated with pain in fibromyalgia and rheumatoid arthritis. & [73] \\
\hline $\begin{array}{l}\text { Mast/stem cell growth factor receptor } \\
\text { Kit }\end{array}$ & Higher levels associated with pain in fibromyalgia and rheumatoid arthritis. & [73] \\
\hline $\begin{array}{l}\text { Prolow-density lipoprotein receptor- } \\
\text { related protein I }\end{array}$ & Higher levels associated with pain in fibromyalgia and rheumatoid arthritis. & [73] \\
\hline Apolipoprotein-CI & Increased levels in neuropathic pain. & {$[74]$} \\
\hline Autotaxin & Increased levels in patients with fibromyalgia. & {$[74]$} \\
\hline Cystatin C & Elevated levels in obstetric patients in labor who were in severe pain. & {$[75]$} \\
\hline Tumor necrosis factor beta (TNF- $\beta$ ) & $\begin{array}{l}\text { Elevated levels in patients with trigeminal neuralgia and decreased after microvascular } \\
\text { decompression surgery towards levels observed in healthy controls. }\end{array}$ & [76] \\
\hline $\begin{array}{l}\text { TNF-related apoptosis inducing ligand } \\
\text { (TRAIL) }\end{array}$ & $\begin{array}{l}\text { Elevated levels in patients with trigeminal neuralgia and decreased after microvascular } \\
\text { decompression surgery towards levels observed in healthy controls. }\end{array}$ & [76] \\
\hline Growth hormone AI (PRL) & Lower expression after burst spinal cord stimulation in patients with neuropathic pain. & {$[72]$} \\
\hline Nucleobindin-2 (NUCB2) & Lower expression after burst spinal cord stimulation in patients with neuropathic pain. & [72] \\
\hline Calbindin (CALBI) & Lower expression after burst spinal cord stimulation in patients with neuropathic pain. & [72] \\
\hline Acyl-CoA binding protein $(\mathrm{CBI})$ & Lower expression after burst spinal cord stimulation in patients with neuropathic pain. & [72] \\
\hline ProSAAS (PCSKIN) & Lower expression after burst spinal cord stimulation in patients with neuropathic pain. & [72] \\
\hline Endothelin-3 (END3) & Lower expression after burst spinal cord stimulation in patients with neuropathic pain. & [72] \\
\hline Cholecystokinin (CCK) & Lower expression after burst spinal cord stimulation in patients with neuropathic pain. & [72] \\
\hline
\end{tabular}


Fibromyalgia is a heterogeneous condition that is characterized by widespread musculoskeletal pain, pain elicited on digital palpation of tender points, fatigue, sleep issues, and mood disorder. Despite decades of research on fibromyalgia, there continues to be limited data on its underlying mechanism of action, lack of diagnostic and prognostic markers, and limited treatment options. Several studies have highlighted measurable changes in CSF biomarkers that may have potential diagnostic and prognostic implications. ${ }^{71,73,74}$ Sarchielli et al studied 16 chronic migraine patients, 15 patients with probable chronic migraine and probably analgesic-abuse headache, 20 fibromyalgia patients, and 20 control patients. ${ }^{71}$ This study reported that compared to healthy control patients, there was lower levels of glial cell line-derived neurotrophic factor (GDNF) and somatostatin (SST). Khoonsari et al measured CSF biomarkers in 13 fibromyalgia patients and 11 rheumatoid arthritis patients, and identified 10 proteins that may be associated with pain including neural cell adhesion molecule L1, complement C4-A, lysozyme C, receptor-type tyrosine-protein phosphatase zeta, apolipoprotein D, alpha-1-antichymotrypsin, granulins, calcium/ calmodulin-dependent protein kinase type II subunit alpha, mast/stem cell growth factor receptor kit, prolow-density lipoprotein receptor-related protein $1 .^{73}$ While the authors postulate that these biomarkers are known to be involved in pain mechanisms, these associations are novel in the context of fibromyalgia. Lind et al studied 40 fibromyalgia patients and 134 control patients, and reported increased levels of autotaxin in fibromyalgia patients. ${ }^{74}$

A unique pre- and post-treatment study design was reported by Ericson et al, in which the levels of $92 \mathrm{CSF}$ protein biomarkers related to inflammation were measured before and after microvascular decompression surgery in trigeminal neuralgia patients. ${ }^{76}$ This study reported that TNF- $\beta$ and TNF-related apoptosis inducing ligand (TRAIL) were elevated at baseline in patients with trigeminal neuralgia and decreased towards levels observed in healthy controls after patients underwent microvascular decompression surgery.

Similarly, a pre- and post-treatment study design was conducted by Royds et al. ${ }^{72}$ This study performed proteomic and neuropeptide CSF analysis in four patients with neuropathic pain who underwent burst spinal cord stimulation. Compared to baseline levels prior to spinal cord stimulator implant, there was decreased expression of growth hormone A1 (PRL), somatostatin (SST), nucleobindin-2 (NUCB2), calbindin (CALB1), acyl-CoA binding protein (DBI), proSAAS (PCSK1N), endothelin-3 (END3) and cholecystokinin (CCK) after 8 weeks of burst spinal cord stimulation therapy.

Finally, Mannes et al studied obstetric patients experiencing severe labor pain and measured CSF levels of cystatin $\mathrm{C}$, which is cysteine protease inhibitor belonging to the cystatin superfamily. ${ }^{75}$ This study included five pregnant females with severe labor pain and five control pregnant patients who were presenting for elective Cesarean section and were not in severe pain. Compared to control patients, pregnant females with severe pain had elevated levels of cystatin $\mathrm{C}$ in the CSF. The study concluded that this observation is consistent with prior animal studies that report persistent pain inducing synthesis of cystatin $\mathrm{C}$ from the dorsal spinal cord and with subsequent overflow of surplus amounts into the CSF.

\section{Salivary Biomarkers}

The study of salivary diagnostic markers is an emerging field using nanotechnology and molecular studies to diagnose oral disease and systemic disorders (Table 5). ${ }^{77-87}$ Salivary biomarkers, along with urinary biomarkers, have the distinct advantage of non-invasive sampling. This makes clinical use of these laboratory studies more comfortable and accessible for patients. ${ }^{88}$ Most biomarkers that are present in blood and urine are also detectable in saliva samples. $^{88}$

Several studies have examined the utility of salivary biomarkers as diagnostic and prognostic measures of pain in fibromyalgia patients. Catley et al compared salivary levels of cortisol, diurnal cycles of cortisol, and the impact of psychological stress on cortisol reactivity in 21 fibromyalgia patients versus 22 healthy control patients. ${ }^{77}$ They reported that higher average levels of salivary cortisol were detected in fibromyalgia patients versus healthy control patients, although there was no difference in the diurnal cycle of cortisol or psychological stress reactivity. However, in chronic manifestations of fibromyalgia and widespread musculoskeletal pain, cortisol levels may actually be decreased with a blunted diurnal slope. This was depicted by Generaal et al which included 471 patients with chronic multisite musculoskeletal pain and 654 control patients, and compared their salivary cortisol levels at awakening, one-hour postawakening, evening, as well as diurnal slopes. ${ }^{78}$ They reported that patients with chronic musculoskeletal pain had lower cortisol levels on awakening and evening 
Table 5 Salivary Biomarkers and Relevance in Chronic Pain Pathology

\begin{tabular}{|c|c|c|}
\hline Biomarker & Relevance & References \\
\hline Cortisol & $\begin{array}{l}\text { Increased in early fibromyalgia and positively associated with pain, stress, and } \\
\text { depression. } \\
\text { Decreased in chronic fibromyalgia. } \\
\text { Increased in dental pain (pulpal or periapical inflammation). } \\
\text { Fluctuates depending on timing of sampling, but may be increased in fibromyalgia. }\end{array}$ & [77-80] \\
\hline Alpha-amylase & $\begin{array}{l}\text { Increased in burning mouth syndrome. } \\
\text { Increased in temporomandibular disorder. }\end{array}$ & {$[81,82]$} \\
\hline Transaldolase & Overexpression in female patients with fibromyalgia. & [83] \\
\hline Phosphoglycerate mutase I & $\begin{array}{l}\text { Increased in fibromyalgia. } \\
\text { Decreased levels after treatment with mud-bath and balneotherapy in fibromyalgia } \\
\text { patients. }\end{array}$ & {$[83,84]$} \\
\hline Calgranulin & Increased calgranulina $A$ and $C$ in fibromyalgia. & [83] \\
\hline Glutamate & Increased in chronic migraine. & {$[85]$} \\
\hline Immunoglobulin A ( $\lg A)$ & Increased in burning mouth syndrome. & {$[81]$} \\
\hline $\begin{array}{l}\text { Macrophage inflammatory protein- } 4 \\
\text { (MIP4) }\end{array}$ & Increased in burning mouth syndrome. & {$[81]$} \\
\hline Uric acid & Decreased in burning mouth syndrome. & {$[81]$} \\
\hline Ferric reducing activity of plasma (FRAP) & Decreased in burning mouth syndrome. & {$[81]$} \\
\hline Nerve growth factor (NGF) & Decreased in temporomandibular disorder. & {$[82]$} \\
\hline $\begin{array}{l}\text { Brain-derived neurotrophic factor } \\
\text { (BDNF) }\end{array}$ & $\begin{array}{l}\text { Decreased in temporomandibular disorder. } \\
\text { Decreased levels after treatment with mud-bath and balneotherapy in fibromyalgia } \\
\text { patients. }\end{array}$ & {$[82,84]$} \\
\hline Interleukin-I beta (IL-I $\beta$ ) & Increased in dental pain (pulpal or periapical inflammation). & [79] \\
\hline Interleukin-6 (IL-6) & Increased in dental pain (pulpal or periapical inflammation). & [79] \\
\hline C-reactive protein (CRP) & Decreased in chronic periodontitis. & {$[86]$} \\
\hline Alpha-2-macroglobulin (alpha-2M) & Decreased in chronic periodontitis. & {$[86]$} \\
\hline Toll-like receptor 2 (TLR-2) & Increased in burning mouth syndrome. & [87] \\
\hline
\end{tabular}

times, as well as a blunted diurnal slope. Some studies have even postulated that despite the chronicity of symptoms in fibromyalgia, there are fluctuating levels of biomarkers even within each day. To investigate these daily fluctuating biomarker levels, Fischer et al conducted a 14-day study on 32 female patients with fibromyalgia who recorded diary entries on momentary stress and pain levels, and collected samples during those respective for measurement of cortisol and alpha-amylase. ${ }^{80}$ The study reported that momentary cortisol was associated with momentary pain, supporting that cortisol may impact diurnal fluctuation of pain levels in fibromyalgia patients.
In addition to cortisol, other notable salivary biomarkers were investigated in fibromyalgia patients. Bazzichi et al conducted a proteomic analysis of saliva in 22 fibromyalgia patients and 26 age- and sex-matched healthy control patients. ${ }^{83}$ This study reported significant overexpression of salivary transaldolase, calgranulin A and C, and phosphoglycerate mutase I. To further assess changes in salivary biomarkers with treatment in fibromyalgia patients, another pre- and post-treatment study by Bazzichi et al measured phosphoglycerate mutase I levels in fibromyalgia patients who received mud-bath therapy $(n=21)$ and balneotherapy $(n=20) .{ }^{84}$ This study reported 
significant reductions in phosphoglycerate mutase I levels after both mud-bath and balneotherapy.

Similar to fibromyalgia, another manifestation of spontaneous pain with hyperalgesia, referred pain, and association with psychological comorbidities like depression and anxiety is temporomandibular disorder (TMD) myalgia. ${ }^{82}$ This leads to chronic masticatory pain and is three times more frequent in females compared to males. ${ }^{82}$ Jasim et al compared salivary samples from 39 patients with TMD and 39 pain-free healthy control patients. ${ }^{82}$ This study reported that TMD patients had both higher salivary and plasma levels of glutamate compared to healthy control patients. In addition, salivary nerve growth factor (NGF) and salivary brain-derived neurotrophic factor were decreased in TMD patients compared to healthy control patients.

Burning mouth syndrome is also a spontaneous pain manifestation characterized by intraoral burning and dysesthesias without any evidence of clinical lesions. ${ }^{89}$ The symptoms are predominantly localized to the tongue and lips, and affected patients commonly have comorbid personality changes and mood disorders. ${ }^{81}$ Lopez-Jornet et al analyzed inflammatory biomarkers in saliva samples from 51 patients with burning mouth syndrome and compared them to saliva samples from 31 healthy control patients. ${ }^{81}$ In addition to increased salivary flow, salivary analysis in patients with burning mouth syndrome revealed increased levels of alpha-amylase, immunoglobulin A (IgA), macrophage inflammatory protein-4 (MIP4) compared to controls. Furthermore, there were decreased levels of uric acid and ferric reducing activity of plasma (FRAP) in patients with burning mouth syndrome versus controls. The authors conclude that burning mouth syndrome may affect inflammatory markers within the saliva and the resulting oxidative stress may correlate with the severity of pain.

Saliva also contains a variety of neuropeptides because of salivary gland innervation from nerves originating from the trigeminovascular system and thus can provide insight into nervous system pathology and various headache disorders. ${ }^{85}$ Nam et al collected salivary samples from 46 females with chronic migraine, 50 females with episodic migraine, and 19 healthy control patients and compared salivary glutamate levels. ${ }^{85}$ Glutamate is known to be one of the most abundant excitatory neurotransmitters present in the central nerve system and may be implicated in the pathogenesis of cortical spreading depression, activation of the trigeminal neuron, and central sensitization..$^{90}$ Mean salivary glutamate levels were significantly higher in the chronic migraine cohort compared to the episodic migraine patients and control patients. The salivary glutamate level in the episodic migraine cohort was only marginally elevated compared to control patients.

Pulpal or periapical inflammation may lead to acute dental pain and may also be associated with increased expression of inflammatory biomarkers in the saliva. Haug et al compared 42 patients with pulpal or periapical inflammation seeking emergency dental treatment and 39 control patients. ${ }^{79}$ After biomarker analysis of unstimulated saliva collection, results demonstrated increased levels of cortisol, IL-1 $\beta$, IL-6, as well as greater salivary flow in patients with pulpal or periapical inflammation versus control patients. Another study by Aurer et al measured inflammatory markers in the saliva from 9 edentulous patients, 10 chronic periodontitis patients, 18 aggressive periodontitis patients, and 14 health control patients. ${ }^{86}$ Decreased levels of C-reactive protein (CRP) and alpha-2-macroglobulin (alpha-2M) were detected in edentulous patients and patients with chronic periodontitis. Thus, this study concludes that a reduction in host responsiveness in secreting inflammatory markers may play a role in the pathology of chronic manifestations of periodontitis.

\section{Summary}

The studies reviewed suggest that specific urinary, serum, cerebrospinal fluid, and salivary biomarkers may help identify those patients at risk of disease development, and function as a prognostic indicator for disease progression and treatment response. However, current individual and panel-based biomarkers have limited usability in regards to informing clinical decision-making at this time.

\section{Future Directions}

There is no doubt that the number of candidate biomarkers for complex disease, including chronic pain, will continue to increase as high throughput 'multi-omics' platforms become commonplace in government, academic, and industry research and development settings. Emerging highefficiency techniques now allow for single-cell analysis and spatial metabolomics/transcriptomics, which will almost certainly further our understanding of the role of specific biomarkers and immune cells in chronic pain disorders.

Successful validation of candidate biomarkers is a rigorous, expensive, and continuous process that requires multiple, carefully designed clinical studies to evaluate performance criteria across the wide spectrum of chronic pain disorders. Throughout the evaluation process, individual candidate biomarkers and biomarker composite 
panels must demonstrate clinical validity and utility if the outcome is to become part of routine clinical care. While the path from biomarker discovery to clinical adoption is often complex, slow, and expensive, few would question the potential impact that robust pain biomarkers would have on the field of pain management.

Additionally, as Backryd described in 2015, subclassification of pain (ie, neuropathic, nociceptive, and nociplastic) and pertinent biomarkers should be an area of focus. ${ }^{91}$ Given that many chronic pain treatments are tailored to specific pain sub-classifications, the ability to collect and monitor unique biomarkers is imperative.

\section{Conclusion}

While the use of objective laboratory measures has become commonplace in other areas of medicine, it has been relatively lacking in the field of chronic pain medicine. Ultimately, a biomarker pain panel that highlights metabolic abnormalities, oxidative stressors, and nutritional deficiencies may help to identify the underlying treatable causes of pain and potentially provide evidence of treatment response. Certainly, additional research is necessary before specific recommendations can be made, but the potential benefits for the treatment of chronic pain make this an important and necessary area of future study.

\section{Funding}

There is no funding to report.

\section{Disclosure}

Dr Jonathan M Hagedorn reports personal fees for consulting and advisory board from Abbott, Boston Scientific, and Nevro, outside the submitted work. Dr Joshua Gunn has a patent "Methods of Diagnosing and Treating Particular Causal Components of Chronic Pain in a Patient" pending to Ethos Laboratories; and Dr Gunn is the co-founder and Chief Scientific Officer of Ethos Research \& Development. Ethos R\&D developed and validated a novel biomarker assay mentioned in this manuscript. Dr Timothy R Deer reports personal fees for consulting from Ethos, during the conduct of the study. The authors report no other conflicts of interest in this work.

\section{References}

1. Reckziegel D, Vachon-Presseau E, Petre B, et al. Deconstructing biomarkers for chronic pain: context and hypothesis dependent biomarker types in relation to chronic pain. Pain. 2019;160(Suppl 1): S37-S48. doi:10.1097/j.pain.0000000000001529
2. Sun AL, Ni YH, Li XB, et al. Urinary methylmalonic acid as an indicator of early vitamin B12 deficiency and its role in polyneuropathy in type 2 diabetes. $J$ Diabetes Res. 2014;2014:1-6. doi:10.1155/2014/921616

3. Langan R, Goodbred A. Vitamin B12 deficiency: recognition and management. Am Fam Physician. 2017;96(6):384-389.

4. Gürsoy AE, Kolukısa M, Babacan-Yıldız G, Çelebi A. Subacute combined degeneration of the spinal cord due to different etiologies and improvement of MRI findings. Case Rep Neurol Med. 2013;2013:1-5.

5. Hammond N, Wang Y, Dimachkie MM, Barohn RJ. Nutritional neuropathies. Neurol Clin. 2013;31(2):477-489. doi:10.1016/j. ncl.2013.02.002

6. Heller CA, Friedman PA. Pyridoxine deficiency and peripheral neuropathy associated with long-term phenelzine therapy. Am $J$ Med. 1983;75(5):887-888. doi:10.1016/0002-9343(83)90422-9

7. Raskin NH, Fishman RA. Pyridoxine-deficiency neuropathy due to hydralazine. N Engl J Med. 1965;273(22):1182-1185. doi:10.1056/ NEJM196511252732203

8. Corken M, Porter J. Is vitamin B 6 deficiency an under-recognized risk in patients receiving haemodialysis? A systematic review: 2000-2010. Nephrology. 2011;16(7):619-625. doi:10.1111/j.1440-1797.2011.01479.x

9. Sande JS, Ulvik A, Midttun Ø, et al. Vitamin B-6 status correlates with disease activity in rheumatoid arthritis patients during treatment with TNF $\alpha$ inhibitors. J Nutr. 2019;149(5):770-775. doi:10.1093/jn/ nxz001

10. Kohlstadt I, ed. Food and Nutrients in Disease Management. 1st ed. Boca Raton: CRC Press; 2009.

11. Kamat PK, Mallonee CJ, George AK, Tyagi SC, Tyagi N. Homocysteine, alcoholism, and its potential epigenetic mechanism. Alcohol Clin Exp Res. 2016;40(12):2474-2481. doi:10.1111/ acer. 13234

12. Morrow LE, Grimsley EW. Long-term diuretic therapy in hypertensive patients: effects on serum homocysteine, vitamin B6, vitamin B12, and red blood cell folate concentrations. South Med J. 1999;92 (9):866-870. doi:10.1097/00007611-199909000-00003

13. Butler B, Acosta G, Shi R. Exogenous Acrolein intensifies sensory hypersensitivity after spinal cord injury in rat. J Neurol Sci. 2017;379:29-35. doi:10.1016/j.jns.2017.05.039

14. Lin Y, Chen Z, Tang J, Cao P, Shi R. Acrolein contributes to the neuropathic pain and neuron damage after ischemic-reperfusion spinal cord injury. Neuroscience. 2018;384:120-130. doi:10.1016/j. neuroscience.2018.05.029

15. Moghe A, Ghare S, Lamoreau B, et al. Molecular mechanisms of acrolein toxicity: relevance to human disease. Toxicol Sci. 2015;143 (2):242-255. doi:10.1093/toxsci/kfu233

16. Kim H, Chen L, Lim G, et al. Brain indoleamine 2,3-dioxygenase contributes to the comorbidity of pain and depressionUEG Week 2013 Oral Presentations. J Clin Invest. 2012;122(8):2940-2954. doi:10.1172/JCI61884

17. Dantzer R, O'Connor JC, Lawson MA, Kelley KW. Inflammationassociated depression: from serotonin to kynurenine. Psychoneuroendocrinology. 2011;36(3):426-436. doi:10.1016/j. psyneuen.2010.09.012

18. Dantzer R. Role of the kynurenine metabolism pathway in inflammation-induced depression: preclinical approaches. In: Current Topics in Behavioral Neurosciences. Vol. 31. Springer Verlag; 2017:117.

19. de Oliveira FR, Fantucci MZ, Adriano L, et al. Neurological and inflammatory manifestations in Sjogren's syndrome: the role of the kynurenine metabolic pathway. Int J Mol Sci. 2018;19(12):3953. doi:10.3390/ijms 19123953

20. Savitz J. The kynurenine pathway: a finger in every pie. Mol Psychiatry. 2020;25(1):131-147. doi:10.1038/s41380-019-0414-4

21. Figueroa-Romero C, Sadidi M, Feldman EL. Mechanisms of disease: the oxidative stress theory of diabetic neuropathy. Rev Endocr Metab Disord. 2008;9(4):301-314. doi:10.1007/s11154-008-9104-2 
22. Darmaun D, Smith SD, Sweeten S, Sager BK, Welch S, Mauras N. Evidence for accelerated rates of glutathione utilization and glutathione depletion in adolescents with poorly controlled type 1 diabetes. Diabetes. 2005;54(1):190-196. doi:10.2337/diabetes.54.1.190

23. Emmett M. Acetaminophen toxicity and 5-oxoproline (pyroglutamic acid): a tale of two cycles, one an ATP-depleting futile cycle and the other a useful cycle. Clin J Am Soc Nephrol. 2014;9(1):191-200. doi:10.2215/CJN.07730713

24. Qu H, Guo M, Chai H, Wang WT, Ga ZY, Shi DZ. Effects of coenzyme Q10 on statin-induced myopathy: an updated meta-analysis of randomized controlled trials. $\mathrm{J}$ Am Heart Assoc. 2018;7(19):e009835. doi:10.1161/JAHA.118.009835

25. Hernández-Camacho JD, Bernier M, López-Lluch G, Navas $P$. Coenzyme Q10 supplementation in aging and disease. Front Physiol. 2018;9(FEB):44. doi:10.3389/fphys.2018.00044

26. Flanagan JL, Simmons PA, Vehige J, Willcox MD, Garrett Q. Role of carnitine in disease. Nutr Metab. 2010;1:7.

27. Miranda-Massari R. Metabolic Correction in the Management of Diabetic Peripheral Neuropathy: improving Clinical Results Beyond Symptom Control. Curr Clin Pharmacol. 2011;6(4):260-273. doi:10.2174/157488411798375967

28. Sforzini L, Nettis MA, Mondelli V, Pariante CM. Inflammation in cancer and depression: a starring role for the kynurenine pathway. Psychopharmacology. 2019;236(10):2997-3011. doi:10.1007/ s00213-019-05200-8

29. Lapin IP, Oxenkrug GF. Intensification of the central serotoninergic processes as a possible determinant of the thymoleptic effect. Lancet. 1969;293(7586):132-136. doi:10.1016/S0140-6736(69)91140-4

30. Martin SL, Power A, Boyle Y, Anderson IM, Silverdale MA, Jones AKP. 5-HT modulation of pain perception in humans. Psychopharmacology. 2017;234(19):2929-2939. doi:10.1007/ s00213-017-4686-6

31. Pertovaara A. Noradrenergic pain modulation. Prog Neurobiol. 2006;80(2):53-83. doi:10.1016/j.pneurobio.2006.08.001

32. Bannister K, Dickenson AH. What do monoamines do in pain modulation? Curr Opin Support Palliat Care. 2016;10(2):143-148. doi:10.1097/SPC.0000000000000207

33. Liang W, Wu Z, Zhang G, et al. A urine-based biomarker for chronic prostatitis/chronic pelvic pain syndrome: a retrospective multi-center study. Transl Androl Urol. 2020;9(5):2218226. doi:10.21037/tau-20-1268

34. Gu CY, Huang YQ, Han CT, et al. Clinical significance of urine prostatic exosomal protein in the diagnosis of prostate cancer. $\mathrm{Am}$ $J$ Cancer Res. 2019;9(5):1074-1078.

35. Roy RA, Stephens AJ, Daisy C, et al. Association of longitudinal changes in symptoms and urinary biomarkers in patients with urological chronic pelvic pain syndrome: a MAPP Research Network Study. J Urol. 2021;205(2):514-523. doi:10.1097/JU.0000000000001391

36. Woodworth DC, Dagher A, Curatolo A, et al. Changes in brain white matter structure are associated with urine proteins in urological chronic pelvic pain syndrome (UCPPS): a MAPP Network study. PLoS One. 2018;13(12):e0206807. doi:10.1371/journal. pone.0206807

37. Dagher A, Curatolo A, Sachdev M, et al. Identification of Novel Non-invasive Biomarkers of Urinary Chronic Pelvic Pain Syndrome (UCPPS): findings from the Multidisciplinary Approach to the Study of Chronic Pelvic Pain (MAPP) Research Network. BJU Int. 2017;120(1):130. doi:10.1111/bju.13832

38. Harpole M, Davis J, Espina V. Current state of the art for enhancing urine biomarker discovery. Expert Rev Proteomics. 2016;13(6):609. doi:10.1080/14789450.2016.1190651

39. Lopez-Gonzalez MJ, Landry M, Favereaux A. MicroRNA and chronic pain: from mechanisms to therapeutic potential. Pharmacol Ther. 2017;180:1-15. doi:10.1016/j.pharmthera.2017.06.001

40. Niculescu AB, Le-niculescu H, Levey DF, et al. Towards precision medicine for pain: diagnostic biomarkers and repurposed drugs. Mol Psychiatry. 2019;24(4):501-522. doi:10.1038/s41380-018-0345-5
41. Choi HY, Lee CH. Can beta-endorphin be used as a biomarker for chronic low back pain? a meta-analysis of randomized controlled trials. Pain Med. 2019;20(1):28-36. doi:10.1093/pm/pny186

42. D'Agnelli S, Arendt-Nielsen L, Gerra MC, et al. Fibromyalgia: genetics and epigenetics insights may provide the basis for the development of diagnostic biomarkers. Mol Pain. 2019;2:15.

43. Calvo M, Davies AJ, Hebert HL, et al. The genetics of neuropathic pain from model organisms to clinical application. Neuron. 2019;104 (4):637-653. doi:10.1016/j.neuron.2019.09.018

44. Gunn J, Hill M, Cotten B, Deer TR. An analysis of biomarkers in chronic pain patients. Pain Physician. 2020;23:23.

45. Amirdelfan K, Pope JE, Gunn J, et al. Clinical validation of a multi-biomarker assay for the evaluation of chronic pain patients in a cross-sectional, observational study. Pain Ther. 2020;9(2):511-529. doi:10.1007/s40122-020-00175-3

46. Peabody J, Paculdo D, Tamondong-Lachica D, Cabaluna IT, Gunn J. Randomized trial on the clinical utility of a novel biomarker panel to identify treatable determinants of chronic pain. Diagnostics. 2020;10 (8):513. doi:10.3390/diagnostics10080513

47. McCloy K, Doan N, Abeyratne U, Systematic A. Review of the association between urinary biomarkers and pain. IFMBE Proc. 2017;63:443-447.

48. Gebhardt K, Brenner H, Sturmer T, et al. The course of high-sensitive C-reactive protein in correlation with pain and clinical function in patients with acute lumbosciatic pain and chronic low back pain - a 6 months prospective longitudinal study. Eur J Pain. 2006;10 (8):711-719. doi:10.1016/j.ejpain.2005.11.005

49. Stürmer T, Raum E, Buchner M, et al. Pain and high sensitivity $\mathrm{C}$ reactive protein in patients with chronic low back pain and acute sciatic pain. Ann Rheum Dis. 2005;64(6):921-925. doi:10.1136/ $\operatorname{ard} .2004 .027045$

50. Zu B, Pan H, Zhang XJ, Yin ZS. Serum levels of the inflammatory cytokines in patients with lumbar radicular pain due to disc herniation. Asian Spine J. 2016;10(5):843-849. doi:10.4184/ asj.2016.10.5.843

51. Gadient RA, Otten UH. Interleukin-6 (IL-6)-a molecule with both beneficial and destructive potentials. Prog Neurobiol. 1997;52 (5):379-390. doi:10.1016/S0301-0082(97)00021-X

52. Cunha FQ, Poole S, Lorenzetti BB, Ferreira SH. The pivotal role of tumour necrosis factor alpha in the development of inflammatory hyperalgesia. Br J Pharmacol. 1992;107(3):660-664. doi:10.1111/ j.1476-5381.1992.tb14503.x

53. De Queiroz BZ, Sirineu Pereira D, Antunes Lopes R, et al. Association Between the plasma levels of mediators of inflammation with pain and disability in the elderly with acute low back pain: data from the Back Complaints in the Elders (BACE)-Brazil study. Spine. 2016;41(3):197-203. doi:10.1097/BRS.0000000000001214

54. Bruunsgaard H, Galbo H, Halkjaer-Kristensen J, Johansen TL, MacLean DA, Pedersen BK. Exercise-induced increase in serum interleukin-6 in humans is related to muscle damage. $J$ Physiol. 1997;499(Pt 3):833-841. doi:10.1113/jphysiol.1997.sp021972

55. Pedersen BK, Steensberg A, Schjerling P. Muscle-derived interleukin-6: possible biological effects. J Physiol. 2001;536(Pt 2):329-337. doi:10.1111/j.1469-7793.2001.0329c.xd

56. Pedersen LM, Schistad E, Jacobsen LM, Roe C, Gjerstad J. Serum levels of the pro-inflammatory interleukins 6 (IL-6) and-8 (IL-8) in patients with lumbar radicular pain due to disc herniation: a month prospective study. Brain Behav Immun. 2015;46:132-136. doi:10.1016/j.bbi.2015.01.008

57. Ostrowski K, Rohde T, Zacho M, Asp S, Pedersen BK. Evidence that interleukin-6 is produced in human skeletal muscle during prolonged running. J Physiol. 1998;508(Pt 3):949-953. doi:10.1111/j.14697793.1998.949bp.x

58. Uçeyler N, Rogausch JP, Toyka KV, Sommer C. Differential expression of cytokines in painful and painless neuropathies. Neurology. 2007;69(1):42-49. doi:10.1212/01.wnl.0000265062.92340.a5 
59. Luchting B, Rachinger-Adam B, Zeitler J, et al. Disrupted TH17/Treg balance in patients with chronic low back pain. PLoS One. 2014;9(8): e104883. doi:10.1371/journal.pone.0104883

60. Cheng L, Fan W, Liu B, Wang X, Nie L. Th17 lymphocyte levels are higher in patients with ruptured than non-ruptured lumbar discs, and are correlated with pain intensity. Injury. 2013;12:1805-1810. doi:10.1016/j.injury.2013.04.010

61. Xue H, Yao Y, Wang X, et al. Interleukin-21 is associated with the pathogenesis of lumbar disc herniation. Iran $J$ Allergy Asthma Immunol. 2015;14(5):509-518.

62. Sowa GA, Perera S, Bechara B, et al. Associations between serum biomarkers and pain and pain-related function in older adults with low back pain: a pilot study. $J$ Am Geriatr Soc. 2014;62 (11):2047-2055. doi:10.1111/jgs.13102

63. Xie P, Liu B, Chen R, et al. Comparative analysis of serum proteomes: identification of proteins associated with sciatica due to lumbar intervertebral disc herniation. Biomed Rep. 2014;2 (5):693-698. doi:10.3892/br.2014.295

64. Licciardone JC, Kearns CM, Hodge LM, Bergamini MV. Associations of cytokine concentrations with key osteopathic lesions and clinical outcomes in patients with nonspecific chronic low back pain: results from the OSTEOPATHIC Trial. J Am Osteopath Assoc. 2012;112(9):596-605. doi:10.7556/jaoa.2012.112.9.596

65. Wang K, Bao JP, Yang S, et al. A cohort study comparing the serum levels of pro- or anti-inflammatory cytokines in patients with lumbar radicular pain and healthy subjects. Eur Spine J. 2016;25 (5):1428-1434. doi:10.1007/s00586-015-4349-4

66. Wang H, Schiltenwolf M, Buchner M. The role of TNF-alpha in patients with chronic low back pain-a prospective comparative longitudinal study. Clin J Pain. 2008;24(3):273-278. doi:10.1097/ AJP.0b013e31816111d3

67. Wang H, Ahrens C, Rief W, Gantz S, Schiltenwolf M, Richter W. Influence of depression symptoms on serum tumour necrosis factor-alpha of patients with chronic low back pain. Arthritis Res Ther. 2010;12(5):R186. doi:10.1186/ar3156

68. Ang DC, Moore MN, Hilligoss J, Tabbey R. MCP-1 and IL- 8 as pain biomarkers in fibromyalgia: a pilot study. Pain Med. 2011;12 (8):1154-1161. doi:10.1111/j.1526-4637.2011.01179.x

69. Brisby H, Olmarker K, Larsson K, Nutu M, Rydevik B Proinflammatory cytokines in cerebrospinal fluid and serum in patients with disc herniation and sciatica. Eur Spine J. 2002;11 (1):62-66. doi:10.1007/s005860100306

70. Wahlbeck K, Sundblom M, Kalso E, Tigerstedt I, Rimón R. Elevated plasma vasopressin and normal cerebrospinal fluid angiotensin-converting enzyme in chronic pain disorder. Biol Psychiatry. 1996;40(10):994-999. doi:10.1016/0006-3223(95) 00577-3

71. Sarchielli P, Alberti A, Candeliere A, Floridi A, Capocchi G, Calabresi P. Glial cell line-derived neurotrophic factor and somatostatin levels in cerebrospinal fluid of patients affected by chronic migraine and fibromyalgia. Cephalalgia. 2006;26(4):409-415. doi:10.1111/j.1468-2982.2005.01048.x

72. Royds J, Conroy MJ, Dunne MR, et al. Examination and characterisation of burst spinal cord stimulation on cerebrospinal fluid cellular and protein constituents in patient responders with chronic neuropathic pain - A Pilot Study. J Neuroimmunol. 2020;344:577249. doi:10.1016/j.jneuroim.2020.577249

73. Khoonsari PE, Ossipova E, Lengqvist J, et al. The human CSF pain proteome. J Proteomics. 2019;190:67-76. doi:10.1016/j.jprot.201 8.05.012

74. Lind AL, Just D, Mikus M, et al. CSF levels of apolipoprotein C1 and autotaxin found to associate with neuropathic pain and fibromyalgia. J Pain Res. 2019;12:2875-2889. doi:10.2147/JPR. S215348
75. Mannes AJ, Martin BM, Yang HT, et al. Cystatin C as a cerebrospinal fluid biomarker for pain in humans. Pain. 2003;102(3):251-256. doi:10.1016/S0304-3959(02)00403-7

76. Ericson H, Abu Hamdeh S, Freyhult E, et al. Cerebrospinal fluid biomarkers of inflammation in trigeminal neuralgia patients operated with microvascular decompression. Pain. 2019;160(11):2603-2611. doi:10.1097/j.pain.0000000000001649

77. Catley D, Kaell AT, Kirschbaum C, Stone AA. A naturalistic evaluation of cortisol secretion in persons with fibromyalgia and rheumatoid arthritis. Arthritis Care Res. 2000;13(1):51-61. doi:10.1002/15290131(200002)13:1<51::AID-ART8>3.0.CO;2-Q

78. Generaal E, Vogelzangs N, Macfarlane GJ, et al. Reduced hypothalamic-pituitary-adrenal axis activity in chronic multi-site musculoskeletal pain: partly masked by depressive and anxiety disorders. BMC Musculoskelet Disord. 2014;15:227. doi:10.1186/ 1471-2474-15-227

79. Haug SR, Marthinussen MC. Acute dental pain and salivary biomarkers for stress and inflammation in patients with pulpal or periapical inflammation. J Oral Facial Pain Headache. 2019;33(2):227-233. doi:10.11607/ofph.2007

80. Fischer S, Doerr JM, Strahler J, Mewes R, Thieme K, Nater UM. Stress exacerbates pain in the everyday lives of women with fibromyalgia syndrome-The role of cortisol and alpha-amylase. Psychoneuroendocrinology. 2016;63:68-77. doi:10.1016/j.psyneu en.2015.09.018

81. Lopez-Jornet P, Felipe CC, Pardo-Marin L, Ceron JJ, Pons-Fuster E, Tvarijonaviciute A. Salivary biomarkers and their correlation with pain and stress in patients with burning mouth syndrome. J Clin Med. 2020;9(4):929. doi:10.3390/jcm9040929

82. Jasim H, Ghafouri B, Gerdle B, Hedenberg-Magnusson B, Ernberg M. Altered levels of salivary and plasma pain related markers in temporomandibular disorders. J Headache Pain. 2020;21 (1):105. doi:10.1186/s10194-020-01160-Z

83. Bazzichi L, Ciregia F, Giusti L, et al. Detection of potential markers of primary fibromyalgia syndrome in human saliva. Proteomics Clin Appl. 2009;3(11):1296-1304. doi:10.1002/prca.200900076

84. Bazzichi L, Da Valle Y, Rossi A, et al. A multidisciplinary approach to study the effects of balneotherapy and mud-bath therapy treatments on fibromyalgia. Clin Exp Rheumatol. 2013;31(6 Suppl 79):S111-20.

85. Nam JH, Lee HS, Kim J, Chu MK. Salivary glutamate is elevated in individuals with chronic migraine. Cephalalgia. 2018;38 (8):1485-1492. doi:10.1177/0333102417742366

86. Aurer A, Jorgić-Srdjak K, Plancak D, Stavljenić-Rukavina A, AurerKozelj J. Proinflammatory factors in saliva as possible markers for periodontal disease. Coll Antropol. 2005;29(2):435-439.

87. Srinivasan M, Kodumudi KN, Zunt SL. Soluble CD14 and toll-like receptor-2 are potential salivary biomarkers for oral lichen planus and burning mouth syndrome. Clin Immunol. 2008;126(1):31-37. doi:10.1016/j.clim.2007.08.014

88. Malamud D. Saliva as a diagnostic fluid. Dent Clin North Am. 2011;55(1):159-178. doi:10.1016/j.cden.2010.08.004

89. Scala A, Checchi L, Montevecchi M, Marini I, Giamberardino MA. Update on burning mouth syndrome: overview and patient management. Crit Rev Oral Biol Med. 2003;14(4):275-291. doi:10.1177/154411130301400405

90. Ramadan NM. Glutamate and migraine: from Ikeda to the $21 \mathrm{st}$ century. Cephalalgia. 2014;34(2):86-89. doi:10.1177/0333102 413499646

91. Backryd E. Pain in the blood? Envisioning mechanism-based diagnoses and biomarkers in clinical pain medicine. Diagnostics. 2015;5 (1):84-95. doi:10.3390/diagnostics5010084 
Journal of Pain Research

Dovepress

\section{Publish your work in this journal}

The Journal of Pain Research is an international, peer reviewed, open access, online journal that welcomes laboratory and clinical findings in the fields of pain research and the prevention and management of pain. Original research, reviews, symposium reports, hypothesis formation and commentaries are all considered for publication. The manuscript management system is completely online and includes a very quick and fair peer-review system, which is all easy to use. Visit http:// www.dovepress.com/testimonials.php to read real quotes from published authors.

Submit your manuscript here: https://www.dovepress.com/journal-of-pain-research-journal 